\title{
Venous Branch Occlusion: What If It Was Not Just Hypertension!
}

\author{
Sidi Dahi, Joumany Brahim Salem, Mehdi Khamaily, Imane Tarib, Rachid Zerrouk, Yassine Mouzari, \\ Fouad El Asri, Karim Reda, Abdelabarre Oubaaz
}

\section{ABSTRACT}

We report the case of a hypertensive patient who has macular edema, secondary to retinal vein occlusions, revealing pigmentary glaucoma.

54-year-old man had a visual impairment in the left eye. In connection with an retinal vein occlusion; the interview reported hypertension and diabetes, The diagnosis of occlusion of the old retinal vein branch associated with pigmentary glaucoma in a strong myopic is made. Before hypertension, the search for glaucomatous ocular hypertonia must be systematic because it is a particular cause of retinal vein occlusions Related to papillary excavation.

Keywords: Pigmentary glaucoma, Pigment-Dispersion syndrome, retinal venous occlusion, arterial hypertension, macular edema.

\section{INTRODUCTION}

Pigmentary glaucoma characterizes glaucomatous optic neuropathy secondary to pigment dispersion syndrome. It represents only 1 to $1.5 \%$ of glaucoma cases. Its association with branch retinal vein occlusion is rarely described. We report the case of a hypertensive patient who presents with macular edema, secondary to a retinal vein branch occlusion, revealing Pigmentary Glaucoma.
Published Online: October 30, 2020

ISSN: $2593-8339$

DOI: $10.24018 /$ ejmed.2020.2.5.520

Sidi Dahi *

Mohamed V Military Hospital, Mohammed V University of Rabat Morocco.

(e-mail: dahisidi2014@gmail.com)

Joumany Brahim Salem

Mohamed V Military Hospital, Mohammed V University of Rabat, Morocco.

Mehdi Khamaily

Mohamed V Military Hospital, Mohammed V University of Rabat, Morocco.

Imane Tarib

Mohamed V Military Hospital, Mohammed V University of Rabat, Morocco.

Rachid Zerrouk

Mohamed V Military Hospital, Mohammed V University of Rabat, Morocco.

Yassine Mouzari

Mohamed V Military Hospital,

Mohammed V University of Rabat,

Marocco.

Fouad El Asri

Mohamed V Military Hospital, Mohammed V University of Rabat, Morocco.

Karim Reda

Mohamed V Military Hospital,

Mohammed V University of Rabat, Morocco.

Abdelabarre Oubaaz

Mohamed V Military Hospital,

Mohammed V University of Rabat,

Morocco.

*Corresponding Author

\section{CASE REPORT}

This is a 54-year-old patient with known hypertension and diabetes under treatment. The patient consults for reduced visual acuity in the left eye. Visual acuity is rated at $16 / 20$ for the right eye and the fingers for the left eye for refraction at -6.50 and 6.00 , respectively. Examination of the anterior segment reveals a very deep anterior chamber, atrophic areas of the pigmented iris epithelium, pigment deposits on the anterior lens capsule. Intraocular pressure is 24-26mmHg. Gonioscopy reveals the concave aspect of the 
base of the iris and pigmented irido-corneal angle graded IIIII according to the Shaffer classification. The fundus shows an aspect of myopic choroidosis on the right. The fundus of the left eye is similar with the addition of venous tortuosity in the temporal territory, with multiple arteriovenous crossing sites. Exudates and areas of macular hemorrhage are also noted (Fig. 1). Fluorescein angiography shows the collateral circulation and diffusion of fluorescein (Fig. 2). The diagnosis of old branch retinal vein occlusion associated with pigmentary glaucoma in severely myopic is made. An update on the rebalancing of diabetes and hypertension (hypertension) is made. The patient is put on hypotonic drugs, then followed for his glaucoma. Treatment of macular edema by intravitreal injection of antiVEGF is performed.

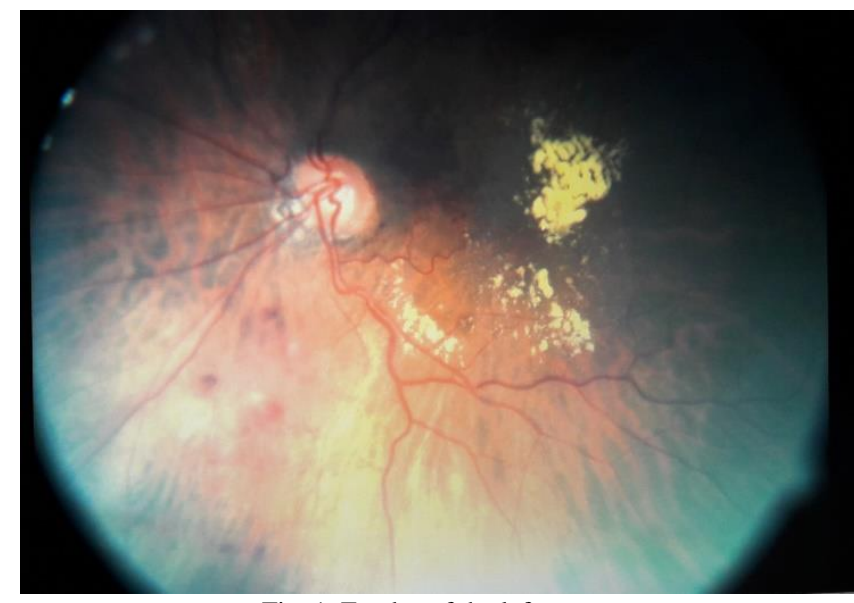

Fig. 1. Fundus of the left eye.

Myopic choroidosis with venous tortuosity in the temporal territory, with multiple arteriovenous crossing sites. Exudates and areas of macular hemorrhage.

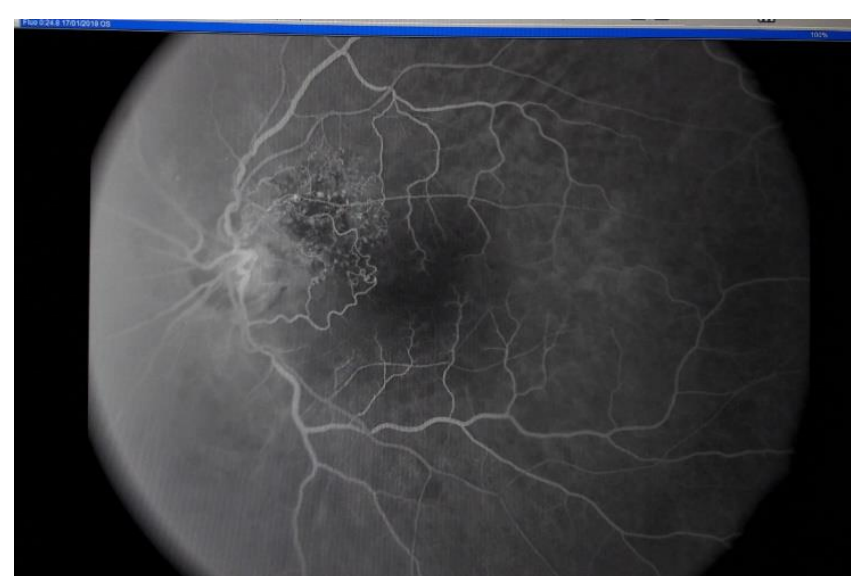

Fig. 2. Fluorescein angiography shows collateral circulation and diffusion of fluorescein.

\section{DISCUSSION}

We can define the retinal venous occlusion as an RVO occurring upstream of a visible arteriovenous crossing. [1]. A special case is represented by the BRVO occurring on a papillary excavation in the glaucomatous, where the site of the occlusion is located within the excavation itself. [2]. Hypertension is the main risk factor for RVO [3]. The pathogenesis of BRVO attributes a causal role to compression of the vein by an arteriosclerotic artery. If the venous obstacle persists long enough, collateral circulation can appear in the form of a venous loop [4]. This allows a retrospective diagnosis of venous occlusion. Pigmentary glaucoma characterizes glaucomatous optic neuropathy secondary to pigment dispersion syndrome. The release of melanin pigment results from rubbing the peripheral region of the posterior epithelium of the iris against the anterior zonular fibers [5]. This friction phenomenon indeed requires a particular ocular anatomical configuration [6]. Typically, PG combines signs of Pigment-Dispersion, pigmentary intraocular hypertonia, structural alterations of the optic nerve, perimetric deficits of a glaucomatous nature [7.8]. Treatment aims to slow down or even stop the process of glaucomatous optic neuropathy [9].

\section{CONCLUSION}

Even in the presence of known hypertension, the search for glaucomatous ocular hypertonia should be part of the systematic assessment, since this is a specific cause of OBVR in connection with papillary excavation.

\section{REFERENCES}

[1] Weger M., Renner W., Steinbrugger I., Cichocki L., Temmel W., Stanger O., and al. Role of thrombophilic gene polymorphisms in branch retinal vein occlusion Ophthalmology 2005; 112: 1910-1915.

[2] Mitchell P., Smith W., Chang A. Prevalence and associations of retinal vein occlusion in Australia. The Blue Mountains Eye Study Arch Ophthalmol Chic Ill 1960 1996; 114: 1243-1247.

[3] Wong T.Y., Larsen E.K.M., Klein R., Mitchell P., Couper D.J., Klein B.E.K., and al. Cardiovascular risk factors for retinal vein occlusion and arteriolar emboli: the atherosclerosis risk in communities \& cardiovascular health studies Ophthalmology 2005; 112: 540-547.

[4] Sperduto R.D., Hiller R., Chew E., Seigel D., Blair N., Burton T.C., and al. Risk factors for hemiretinal vein occlusion: comparison with risk factors for central and branch retinal vein occlusion: the eye disease case-control study Ophthalmology 1998; 105: 765-77.

[5] Green WR, Chan CC, Hutchins GM, Terry JM. Central retinal vein occlusion: a prospective histopathologic study of 29 eyes in 28 cases. Retina 1981; 1: 27-55.

[6] Weinberg D, Dodwell DG, Fern SA. Anatomy of arteriovenous crossings in branch retinal vein occlusion. Am J Ophthalmol 1990; 109: 298-302.

[7] McLeod D. Central retinal vein occlusion with cilioretinal infarction from branch flow exclusion and choroidal arterial steal. Retina 2009; 29: $1381-95$

[8] Sperduto RD, Hiller R, Chew E, et al. Risk factors for hemiretina vein occlusion: comparison with risk factors for central and branch retinal vein occlusion: the eye disease case-control study. Ophthalmology 1998; 105: 765-71.

[9] Rath EZ, Frank RN, Shin DH, Kim C. Risk factors for retinal vein occlusions. A case-control study. Ophthalmology 1992; 99: 509-14. 